\title{
Le transnationalisme en action : le cas des Turcs en
} Suisse

Bülent Kaya et Simone Baglioni

\section{(2) OpenEdition}

Édition électronique

URL : https://journals.openedition.org/remi/4632

DOI : $10.4000 /$ remi.4632

ISSN : $1777-5418$

Éditeur

Université de Poitiers

\section{Édition imprimée}

Date de publication : 1 novembre 2008

Pagination : 167-191

ISBN : 978-2-911627-49-0

ISSN : 0765-0752

Référence électronique

Bülent Kaya et Simone Baglioni, « Le transnationalisme en action : le cas des Turcs en Suisse », Revue européenne des migrations internationales [En ligne], vol. 24 - $n^{\circ} 2$ | 2008, mis en ligne le 01 novembre 2011, consulté le 14 avril 2022. URL : http://journals.openedition.org/remi/4632 ; DOI : https://doi.org/ $10.4000 /$ remi.4632 


\title{
Le transnationalisme en action : le cas des Turcs en Suisse
}

\author{
Bülent KAYA* et Simone BAGLIONI**
}

Le transnationalisme a été également nommé «intégration simultanée (simultaneous embeddedness) dans plus d'une société »(Glick Schiller et al., 1995). Il caractérise désormais les expériences et la vie de presque tous les immigrants. Le quotidien des migrants oscille en permanence entre pays d'origine, pays d'accueil et d'autres endroits où se trouvent des parents, des connaissances des intérêts matériels et symboliques. Une telle relation dynamique entre personnes et lieux géographiques peut être simplement mentale, mais aussi matérielle. En d'autres termes : le transnationalisme peut affecter l'évolution de la vie professionnelle, l'engagement politique aussi bien que les activités socio culturelles ${ }^{1}$.

Parfois, ce caractère national peut ne concerner que des aspects de la vie quotidienne, tels que l'achat dans les épiceries spécialisées en denrées alimentaires du pays d'origine ou la lecture des journaux publiés dans ce pays. Cependant, ces façons multiples et transnationales de percevoir et d'organiser la vie privée peut revêtir des dimensions plus vastes et plus publiques, telles que militer dans ou faire campagne pour un parti politique ou un mouvement social, ce qui revient à agir simultanément dans plus d'un pays. Ou bien encore faire des affaires et promouvoir les échanges économiques entre pays, ou aider des personnes dans le besoin par des actions transnationales.

* Forum suisse pour l'étude des migrations et de la Population (SFM), Université de Neuchâtel, Suisse, SFM@UNINE.CH; WWW.MIGRATIONS-POPULATION.CH

** Forum suisse pour l'étude des migrations et de la Population (SFM), Université de Neuchâtel, Suisse, SFM@UNINE.CH; WWW.MIGRATIONS-POPULATION.CH

1 Le présent article fait partie d'une recherche comparative plus vaste sur « Les pratiques du transnationalisme par les Migrants en Europe de l'Ouest », financée par la Fondation européenne de la Science (ESF) et coordonnée par le Forum suisse pour l'étude des Migrations et de la Population, de l'Université de Neuchâtel. 
Bien que les recherches sur les pratiques transnationales des immigrants puissent concerner plusieurs disciplines des sciences sociales, les études transnationales sont un phénomène très récent en Suisse, et la littérature sur ce thème est très réduite. Le fait que les migrations soient influencées par la circularité de ses mouvements et l'usage de ses liens avec la « diaspora » (Fibbi, 2004 ; Fibbi et Meyer, 2002) ne fut « découvert » qu'à l'extrême fin des années 1990. En dépit d'une longue tradition de «population flottante » en Suisse, le fait que la relation spatiale entre la zone d'origine et les lieux de résidence n'ait provoqué qu'un nombre très limité de nouvelles problématiques de recherche est peut-être dû à la prédominance du dogme de l'intégration qui se fit jour durant la même période (D'Amato et Gerber, 2005).

Les premières études portant sur le transnationalisme au sens vrai furent le reflet des questions politiques d'appartenance, et le fait que des arrangements institutionnels étaient en cours, afin de s'adapter, et d'adapter la situation sociale des migrants (D'Amato, 1997 ; Wicker 2001). Très tôt, un autre courant de recherches se consacra à l'historiographie des expatriés en Suisse, afin d'établir le lien entre leur pays d'origine et leurs droits et devoirs en Suisse (Argast et al., 2003). Les questions économiques furent très vite organisées sur le mode transnational. En particulier, le commerce ethnique offre un accès aisé aux fournisseurs, clients et employés à l'étranger, et minimise par conséquent d'importants coûts intermédiaires. Il offre aussi un support aux communautés transnationales en fournissant des services tels que journaux ou nourriture ethnique, mais aussi en servant de point de rencontre entre expatriés ou en proposant un travail temporaire aux immigrants récents (Piguet, 1999; Riaño, 2003).

Depuis très peu d'années, les études de cas ethnographiques et sociologiques sur le transnationalisme étudient les différentes réalités de l'immigration en Suisse. On mentionnera d'abord le travail sur les réseaux personnels des migrants albanais venus de l'ex-Yougoslavie, une analyse de réseau à l'intérieur du cadre plus vaste des études démographiques (Dahinden, 2005). D'autres recherches furent consacrées à une communauté étrangère plus récente : la nouvelle immigration « arménienne » en Suisse et en Russie, et ses relations aux diasporas classiquement installées (Bachmann et al., 2004). Une approche nouvelle et plus spécifique concerne les mouvements transnationaux africains, en particulier ceux de footballeurs s'installant en Suisse (Poli, 2004).

Bien qu'il existe, dans le paysage social suisse, un intérêt important pour les activités transnationales des immigrants, la recherche empirique est plutôt limitée. Il y a peu de descriptions systématiques des pratiques transnationales des immigrés, qui permettraient de comprendre pourquoi certains d'entre eux décident d'investir leur temps, leurs ressources matérielles et morales dans le but d'établir des liens et des réseaux entre pays. Pourquoi certains préfèrent-ils centrer leur action dans la sphère économique, alors que d'autres préfèrent travailler dans le champ politique ou social ? Quels sont les facteurs ou les caractéristiques qui rendent compte de ces différences? Pendant quelle phase du processus migratoire les immigrés développent-ils des activités transnationales ? Quelles sont leurs motivations à devenir des acteurs transnationaux ? Quel rôle joue le projet migratoire individuel (retour ou intégration) dans la mise en œuvre d'activités transnationales? 
Nous souhaitons contribuer à combler ce manque en nous centrant sur certaines des bases du transnationalisme : un compte-rendu ethnographique des activités transnationales des immigrants turcs ${ }^{2}$. Notre étude explore et s'efforce d'identifier les principaux modèles d'activité transnationale et de proposer des hypothèses explicatives des facteurs politiques, sociaux et économiques qui conditionnent la création et la reproduction d'activités transnationales. Nous nous référons à la définition de Portes des activités transnationales, basée sur une typologie des acteurs : seules les activités crées et mises en œuvre par des acteurs non-institutionnels, qu'il s'agisse de groupes organisés ou de réseaux transfrontaliers d'individus (Portes, 2001) seront considérées comme transnationales.

Les résultats que nous présentons sont basés sur une série d'entretiens semistructurés avec des acteurs transnationaux engagés et des personnages-clés de la communauté turque. Nous avons enquêté sur les pratiques transnationales en rencontrant et en interrogeant : a) une douzaine de personnages-clés qui, par leur implication dans la communauté turque, pouvaient apporter des informations pertinentes sur les pratiques de la communauté ; b) des immigrés de première et de deuxième générations directement engagés dans des activités transnationales. Les entretiens furent conduits à Berne et à Bâle, deux villes accueillant une communauté turque considérable. Dans cet article, nous discutons les expériences transnationales des immigrés turcs dans les domaines économique, politique et socio-culturel, en utilisant les variables temps et étendue pour mesurer l'institutionnalisation et la force des liens transnationaux (Portes et al., 1999 ; Van Amersfoort et al., 1984). Dans chaque domaine, une attention particulière sera apportée à la description et au type d'activité transnationale, aux motivations des acteurs aux critères d'accès, aussi bien qu'aux modèles transnationaux de ces activités. Nous conclurons notre analyse en donnant des aperçus substantiels des principales caractéristiques des pratiques transnationales des immigrés turcs.

\section{PRATIQUES ÉCONOMIQUES TRANSNATIONALES}

Un modèle statique, fondé sur une économie mixte a façonné le marché turc pendant des décennies. La libéralisation de l'économie turque ne commença qu'au cours des années 1980, et s'accompagna d'une ouverture financière centrée d'abord sur le commerce international (Hansen et Cardenas, 1988). Ce fut l'époque de grandes opportunités pour les personnes qui souhaitaient devenir entrepreneurs, ainsi qu'un point de départ pour une nouvelle culture entrepreneuriale. Ce processus donna lieu à un accroissement significatif de la coopération et des relations économiques, en particulier l'import-export de produits et services entre la Turquie et plusieurs payshôtes en Europe, et fit entrer dans le circuit un nombre croissant de Turcs vivant à l'étranger. Durant ces années, quelques immigrés turcs connurent le succès économique, en Allemagne notamment (Piguet, 1999).

2 Nous tenons à souligner que, lorsque nous parlons de "communauté turque », des "Turcs » ou des «immigrés turcs », nous nous référons au critère de la nationalité, et non à l'origine ethnique des personnes qui viennent de Turquie. 
Mais, à côté du dynamisme et des opportunités dans les affaires, offerts par le contexte économique turc, il est également important d'évaluer jusqu'à quel point les immigrés turcs pâtissent du manque de qualification et de discriminations sur le marché du travail (Fibbi et al., 2003) et quel rôle cela joue dans la stimulation de l'activité économique transnationale. En d'autres termes, existe-t-il des facteurs, dans les pays d'accueil, qui stimulent ces activités?

\section{Description et types d'activités}

Selon les experts et les acteurs transnationaux interrogés, il existe une très grande variété d'activités économiques transnationales (à la fois lucratives et sans but lucratif) parmi les immigrés et réfugiés turcs. Nous avons rassemblé ces activités en quatre catégories, selon le commun dénominateur de leurs objectifs : a) activités orientées vers la solidarité ; b) activités orientées vers l'investissement ; c) affaires au sens traditionnel; et enfin d) affaires ethniques (dans une logique de réseau transnational).

\section{Les activités orientées vers la solidarité}

Perçus et conduits dans une logique de solidarité, les envois d'argent et les donations financières aux fonds collectifs sont inclus dans cette catégorie d'activités. Ce sont les activités économiques transnationales les plus traditionnelles et les plus répandues au niveau individuel, les envois d'argent sont effectués pour aider les membres de la famille et/ou des proches. Presque tous les acteurs transnationaux turcs interrogés ont ces pratiques.

Dans certains cas, il s'agit d'améliorer les infrastructures familiales d'habitation, ou d'aider des membres proches de la famille. Cette pratique est surtout le fait des immigrés de la première génération. Ceux de la seconde y sont aussi impliqués, mais de façon plus réduite, et dans la mesure où la famille n'est pas complètement réunie dans le pays-hôte. À la différence de ceux de la première génération, ceux de la seconde envoient très rarement de l'argent à leurs proches en Turquie. D'autre part, la seconde génération semble plus intéressée que la première par les dons aux fonds collectifs. Collectés par des associations ou des fondations dans le cadre d'activités socio culturelles, ces fonds peuvent être considérés comme une espèce particulière de contribution au développement et envoyés au village ou à la ville dont leurs parents sont originaires. Ils ont pour but premier d'améliorer les infrastructures publiques. Cette forme d'activité économique est surtout menée par la seconde génération sur la base du volontariat.

\section{Les activités orientées vers l'investissement}

Certaines activités économiques des immigrés et réfugiés turcs prennent la forme d'investissements. Il faut signaler que les deux générations achètent des biens en Turquie. Pour la première, gagner de l'argent et préparer le retour au pays constituent la principale raison de ces opérations. La seconde génération est motivée par les prix 
peu élevés de maisons de vacances où ils peuvent passer leurs congés d'été avec leur famille. La création d' « entreprises d'immigrés » (connues en Turquie sous le nom de « işçi şirketleri », ou compagnies créées par des émigrants) représenta la plus ancienne modalité collective utilisée par les immigrés pour investir leur argent en Turquie. Ils y ont créé plusieurs compagnies destinées à multiplier le nombre d'usines de production. Pour de nombreuses raisons, en particulier la fraude, ces entreprises n'ont que peu de succès et font souvent, de la part des immigrés, l'objet de soupçons envers ce type d'investissement. Elles attirent cependant une petite minorité de la première génération. Une autre forme d'investissement est le petit commerce (vente, production, hôtel, etc), avec l'aide de membres proches de la famille ou d'amis venus de Turquie. Dans ce cas, les membres des première et deuxième générations investissent leur argent, mais ne s'impliquent pas personnellement dans la gestion, à moins que l'activité ne soit en rapport avec le marché suisse. Pratiquée pour l'essentiel par des membres de la première génération, la participation à des fonds collectifs d'investissement représente une autre façon d'investir. Ce sont en général des compagnies du pays d'origine qui gèrent ces fonds. Collecter des fonds se fait par des moyens informels, à travers des réseaux religieux et sociaux, par exemple.

\section{Les affaires au sens classique (dans une logique transnationale)}

Un certain nombre d'activités économiques des immigrés et réfugiés sont le reflet d'une logique transnationale, au vrai sens du terme. Les personnes interrogées mettent en avant quatre types d'activité. Le premier regroupe des activités économiques dans le domaine du tourisme et des loisirs, comme des compagnies aériennes (des charters créés par les immigrés), des agences de tourisme et tours organisés, et l'organisation d'évènements culturels (concerts). Le second comporte des compagnies d'import-export pour toutes sortes de produits ou de services (différentes formes d'acheminement vers le pays). Ces activités ne sont pas nécessairement limitées à la Turquie et à la Suisse, mais impliquent souvent d'autres pays d'accueil, comme l'Allemagne et l'Italie. Quelques activités économiques consistent à transférer au pays d'origine le capital humain acquis en Suisse ; connaissances, compétences et diverses formes d'expertise (par exemple ouverture d'un centre de psychothérapie, d'un cours de yoga, création d'un vignoble, etc). Par opposition avec les deux premiers types d'activité, pratiquées par tous indépendamment de leur modèle de migration, (forcée ou économique) et de leur appartenance générationnelle (première ou seconde génération), ce type d'activité est plutôt le fait d'anciens réfugiés ou de membres de la seconde génération.

\section{Le « business ethnique » (dans une logique transnationale)}

On peut qualifier de «business ethnique » une dernière catégorie d'activités, telles que le commerce d'approvisionnement, de vente, de loisirs et de production (nourriture, döner kebab, par exemple). Basées surtout dans le pays d'accueil, elles sont menées à l'intérieur d'une logique de réseau transnational. Cela signifie que, dans la plupart des cas, elles n'ont pas beaucoup de rapports avec le pays d'origine, et pour cette raison, ne peuvent être considérées comme transnationales au sens vrai du terme. 
Pourtant, elles renforcent et stimulent les activités économiques transnationales dans la mesure où elles prennent place dans la chaîne des réseaux transnationaux (par exemple, l'utilisation d'épices et autres condiments importés de Turquie pour la production de döner kebab en Suisse). Comme le montre le tableau 1, les activité économiques transnationales des immigrés turcs en Suisse sont très variées, et incluent aussi des activités non lucratives telles que les envois des fonds solidaires adressés aux membres des familles restés dans le pays d'origine.

Tableau 1 : Activités économiques des immigrés turcs en Suisse

\begin{tabular}{|c|c|}
\hline Catégories & Pratiques \\
\hline Orientées vers la solidarité & $\begin{array}{l}\text { Envoi de fonds } \\
\text { Aide financière à des fonds collectifs }\end{array}$ \\
\hline Orientées vers l'investissement & $\begin{array}{l}\text { Achat de biens immobiliers } \\
\text { Création d'« entreprises d'émigrés » } \\
\text { Petit commerce (de détail, hôtels, etc.) } \\
\text { Fonds d'investissements collectifs }\end{array}$ \\
\hline $\begin{array}{l}\text { Affaires } \\
\text { (logique transnationale) }\end{array}$ & $\begin{array}{l}\text { Tourisme et loisirs (agences de voyage, } \\
\text { compagnies musicales, concerts, etc) }\end{array}$ \\
\hline $\begin{array}{l}\text { «thnic Business » qui stimule } \\
\text { les affaires transnationales }\end{array}$ & $\begin{array}{l}\text { Fournitures de biens et services, petit commerce, } \\
\text { loisirs et production dans le pays d'accueil }\end{array}$ \\
\hline
\end{tabular}

Sur la base des activités transnationales présentées ci-dessus, on pourrait se demander pourquoi les immigrés turcs sont aussi motivés à devenir actifs sur le plan transnational, et comment ils accèdent à ces activités.

\section{Motivations et modes d'accès à l'activité économique}

Dans un contexte migratoire, ce sont les circonstances qui dictent les actions en vue de la réussite économique, qui, à la fin, correspondent à leur projet migratoire. C'est particulièrement vrai des immigrés venus en raison des difficultés économiques dans leur pays. Mais au-delà, il est intéressant de se demander pour quelles autres raisons ces immigrés s'impliquent dans une activité transnationale. De quelle manière conçoivent-ils leurs engagements ? A quel moment de leur parcours migratoire choisissent-ils ces activités et de quelle manière y parviennent-ils ? Notre analyse des entretiens avec des acteurs économiques montre que l'engagement dans des activités transnationales est lié à des stratégies à long terme visant à une intégration économique. Yücel ${ }^{3}$, entrepreneur de 45 ans, vit depuis 22 ans en Suisse, importe d'Allemagne de la bijouterie fabriquée en Turquie, et dirige une bijouterie en Suisse. Il confirme cette affirmation :

3 Un grand nombre des personnes interrogées souhaitant rester anonymes, nous avons substitué des noms fictifs aux noms réels. 
"La principale raison qui m'a poussé à entreprendre cette activité était de préparer mon avenir professionnel, afin de m'assurer une vie confortable. »

Au-delà de cette motivation fréquente, parvenir à sortir des difficultés d'entrée sur le marché du travail (par exemple : manque de qualification ou discrimination) constitue une partie décisive de la stratégie de certains acteurs. Erol, 49 ans, habite en Suisse depuis 1994, et distribue des produits textiles importés de son pays en Suisse. Il précise :

«J'ai rencontré beaucoup de difficultés sur la voie de mon intégration professionnelle. Je n'ai ni étudié en Suisse, ni acquis aucune expérience professionnelle reconnue dans ce pays. De plus, il était très difficile pour un étranger de trouver un emploi. Je me suis donc interrogé : comment assurer mon avenir par l'autoemploi?»

Pour d'autres, une activité transnationale peut même être considérée comme la recherche d'une stabilité professionnelle, afin d'éviter le chômage. C'est le cas d'Ahmet, un réfugié de 43 ans, qui travaille et vit en Suisse depuis près de 20 ans. Il a songé à une activité transnationale : la production et l'exportation de döner kebab :

«Au début, je n'avais aucun projet commercial. Je travaillais chez un boucher, ce qui ne me convenait pas. Je devais soit chercher un autre travail, soit être au chômage, situation que j'ai déjà vécue. C'était très dur pour moi. J'ai remarqué que la production de kebab semblait être un domaine très prometteur. J'ai donc décidé d'essayer, et maintenant, ça marche très bien. »

Qu'elles soient naturalisées ou non, immigrées ou réfugiées, les personnes interrogées se considèrent «bien intégrées ». Elles reconnaissent que leur activité transnationale n'a démarré qu'après avoir acquis un certain capital social dans le pays d'accueil, comme les connaissances linguistiques, ce qui est la « source du courage » pour Yücel :

"Je n'ai ni étudié ni acquis de métier en Suisse. Mais j"ai travaillé et appris plusieurs langues dans mon travail. J'ai très vite compris que, pour réussir dans cette société, je devais apprendre des langues. À présent, je possède la nationalité suisse et je me sens très bien intégré, à la fois sur les plans professionnel et social. Je gagne bien ma vie et j'ai plus d'amis suisses que turcs. »

De plus, être impliqué dans des activités transnationales requiert un certain nombre de ressources. Les acteurs économiques transnationaux ont très souvent l'appui matériel ou immatériel de leur famille, d'amis proches ou de leur communauté en Suisse, ce qui leur permet de débuter leur activité, comme en témoigne Küsne, 36 ans, entrepreneur transnational qui vit en Suisse depuis 8 ans. Nous l'avons interrogé sur ses activités socio culturelles :

"Presque toute ma famille est ici. Quand je suis arrivé en Suisse, ma famille m'a aidé à mettre en route mon entreprise. J'ai reçu beaucoup d'appuis parce que ma famille travaillait déjà ici. Il existe aussi des associations où l'on peut rencontrer des gens qui sont toujours prêts à vous aider. Il y a un fort sentiment de solidarité. » 
D'autre part, le transfert des expériences économiques transnationales ou des connaissances acquises dans un autre pays d'accueil peuvent constituer une contribution stimulante pour lancer sa propre activité transnationale, comme dans le cas de Yücel :

"Des cousins de ma femme faisaient le même travail en Allemagne. Ils m'ont suggéré d'entreprendre cette activité. Même, alors que je n'avais pas les ressources financières nécessaires, ils m'ont procuré des marchandises, et ils m'ont aidé à acquérir les connaissances nécessaires pour les affaires. »

Pour Erol, ce qui a facilité son activité actuelle, ce sont ses expériences précédentes et ses contacts dans le pays d'origine :

Q: Pourquoi avoir choisi de travailler dans le textile?

$R$ : J'avais déjà travaillé ans ce secteur avant de venir en Suisse. Je connais beaucoup de marchands qui étaient prêts à m'aider.

$Q$ : Quelle sorte d'appui avez-vous reçu de ces personnes?

$R$ : Pas une aide financière. Mais ils m'ont mis en relation avec des entrepreneurs de haute couture qu'ils connaissaient. Ils m'ont apporté leur garantie auprès de mon premier fournisseur, par exemple.

$Q$ : Pensez-vous que vous auriez réussi sans vos contacts en Turquie?

$R$ : Peut-être. Mais cela aurait été très difficile parce que je n'avais pas assez de capital pour démarrer ma propre affaire. Pour faire des affaires en Turquie, vous avez besoin de capital, de connaissances, d'amis etc. dans le domaine où vous voulez travailler.

Pour la plupart des entrepreneurs transnationaux, les motivations économiques (un avenir meilleur) sont prédominantes. Cela n'a rien de surprenant, et correspond à la logique des affaires. Il est très intéressant d'observer que certains immigrés deviennent actifs sur le plan transnational à la suite d'une stratégie individuelle, destinée à faire face aux difficultés d'insertion et à la discrimination auxquelles ils sont confrontés. Les immigrés sont conscients du fait que le manque de qualification professionnelle pourrait nuire à leur stabilité professionnelle. C'est pourquoi certains anticipent le danger potentiel et s'efforcent de devenir actifs dans le domaine transnational. Il est évident que ce ne sont pas tous les immigrés qui ont ces motivations. Ils ont aussi besoin d'une certaine quantité de « capital » pour accéder aux affaires économiques transnationales. Ce « capital » nécessaire est une combinaison d'une variété de ressources : matérielles (par exemple, de l'argent à investir), culturelles (capacités linguistiques, expérience des affaires...) aussi bien que de ressources sociales (réseaux et contacts familiaux et sociaux). Accéder à ces ressources prend du temps, de l'énergie et de l'investissement de la part de l'immigré.

\section{Caractéristiques des activités transnationales}

Quatre types principaux de liens entre deux pays (d'accueil, d'origine ou pays tiers) caractérisent les activités économiques transnationales. D'abord, la plupart des entrepreneurs mentionnent qu'ils sont en contact avec leurs fournisseurs grâce aux moyens de communication modernes. Cela n'a rien de surprenant, puisqu'ils doivent négocier, recevoir ou placer une commande, comme le souligne Yücel : 
"Je contacte aussi mes fournisseurs par téléphone, fax ou courrier pour envoyer ma liste de commandes. Parfois, nous recevons des produits par la poste. »

Autre forme de communication, plus habituelle et plus nécessaire : les voyages d'affaires. Erol s'envole vers la Turquie près de dix fois par an, pour forger de nouveaux liens commerciaux et pour alimenter ses activités économiques :

"Il est très important que j'aie un accès direct aux produits. Je vais donc souvent à Istanbul. Le face à face avec mes fournisseurs est également très important. Le voyage par avion est si peu cher que je préfère y aller moi-même. Les coups de téléphone, le courrier électronique, etc. sont aussi nécessaires. »

Pendant leurs voyages d'affaires dans les autres pays, les acteurs transnationaux ne rencontrent pas seulement leurs fournisseurs, mais aussi leurs contacts commerciaux potentiels pour explorer de nouvelles niches commerciales en Suisse et en Turquie. Pour ceux qui importent des produits de Turquie, il est essentiel d'avoir aussi des contacts avec les transporteurs en Turquie, afin d'organiser le transport des produits. Enfin, tous mentionnent les transferts financiers commerciaux et l'envoi d'argent aux familles, par le biais d'un système bancaire institutionnalisé en Suisse.

D'autre part, le caractère transnational des activités ne se limite pas aux pays d'origine et d'accueil. Yücel explique :

"Mes activités ne sont pas seulement indirectement liées à mon pays. C'est plutôt avec l'Allemagne que nous avons des relations d'affaires. Bien que les produits soient fabriqués en Turquie, c'est la compagnie de représentation basée en Allemagne qui est responsable des commandes et de leur acheminement vers le reste de l'Europe. Je vais en Allemagne 6 ou 7 fois par an, soit plus souvent qu'en Turquie.»

Le rôle important joué par un autre pays d'accueil, dans ce cas l'Allemagne, dans les activités économiques transnationales, est confirmé par Ahmet :

"J'achète la viande en Suisse, et j'importe les épices de Turquie. Mais nous importons tout l'équipement technique d'Allemagne. Comme le secteur des döner kebab est très développé en Allemagne, j’y vais régulièrement. Je ne vais pratiquement jamais en Turquie pour mes affaires. »

Il est évident que les quatre formes de relations mentionnées ne sont pas spécifiques aux activités économiques transnationales de nos acteurs. Cela correspond aux modèles habituels pratiqués par tous les acteurs économiques transnationaux. Nous pouvons également dire que ces activités fonctionnent selon la logique économique globale, adaptée à leurs propres transactions. On peut décrire le modèle des activités économiques transnationales des immigrés comme l'utilisation systématique et stratégique de leurs contacts et ressources familiales au profit de leurs activités. En d'autres termes, ils mobilisent non seulement leur capital financier, mais aussi leurs réseaux sociaux, familiaux et communautaires dans les pays d'origine pour leurs activités économiques transnationales. 


\section{PRATIQUES TRANSNATIONALES SOCIO-CULTURELLES}

Selon les enquêtes et données statistiques disponibles, les immigrés turcs en Suisse font apparaître un déficit considérable d'intégration, mesuré par des indicateurs tels que les performances scolaires de la seconde génération, les connaissances linguistiques et la sécularisation de la vie culturelle. C'est le cas dans de nombreux pays d'accueil. Certains observateurs comme Tribalat (1995), se référant au contexte français, concluait que les immigrés turcs représentent un cas de retrait identitaire très clair. Cela signifie que les Turcs paraissent s'identifier fortement à leurs liens familiaux, sociaux et culturels, sans adopter les pratiques socio culturelles du pays d'accueil. Il en résulte qu'ils sont plus portés à développer des activités sociales et culturelles intra communautaires. Nous ne discuterons pas la pertinence de cette assomption dans le cas suisse. Nous nous centrerons plutôt sur la dimension transnationale des activités socio culturelles. En d'autres termes, nous analyserons pourquoi et comment les activités culturelles des immigrés turcs s'organisent d'une frontière à l'autre.

\section{Description et types d'activité}

Les activités socio culturelles concernent tous les aspects de la vie sociale. Elles se déroulent à travers un réseau des relations sociales qui environne les immigrés et les réfugiés. Ces activités, nombreuses et variées, peuvent se diviser en cinq types : a) activités quotidiennes ; b) activités caritatives ; c) activités de loisir ; d) activités intellectuelles ; e) activités religieuses.

\section{Activités quotidiennes}

Certaines activités sont quotidiennes, telles que lire le journal, regarder la télévision ou écouter des programmes radio du pays d'origine via le satellite, mais aussi surfer sur internet, téléphoner dans la langue d'origine avec des membres de la famille ou des amis en Turquie ou en Suisse. Grâce à l'évolution des moyens de communication et des transports de technologies, ces activités sont devenues plus facilement accessibles.

\section{Activités caritatives}

Les experts interrogés ont observé une institutionnalisation de ces activités. Il y a une décennie, immigrés et réfugiés turcs mirent en place des fondations pour mener les activités caritatives. Cette tendance n'exclut pas les structures traditionnelles, telles que les associations caritatives ou les engagements individuels ou de petits groupes. Les anciens réfugiés et les immigrés de seconde génération jouent en général un rôle important dans la mise en place de structures institutionnalisées. Selon ces experts, cela tient au fait que certains d'entre eux ont plus de ressources et de compétences que ceux des premières générations (savoir-faire, connaissances linguistiques, réseaux), qui leur permettent d'organiser ces activités. 
Un certain nombre de fondations ont été créées dans le pays d'accueil en tant que sections ou prolongements de fondations pré existantes en Turquie. Leur but principal est d'aider, de différentes façons (financière, logistique et formation), les activités des fondations premières en Turquie. Comme le montre le site internet de la section suisse d'une fondation turque :

"La Fondation Basak est une organisation caritative destinée aux réfugiés et aux enfants des rues. Elle aide ces enfants à s'organiser un projet de vie dans leur propre pays. Outre la protection contre les violences psychologiques et physiques, la Fondation Basak promeut aussi une formation professionnelle pour les enfants des rues. » ${ }^{4}$

Ces fondations sont également utilisées pour organiser des activités de solidarité spécialement destinées aux villages d'origine des immigrés. Elles organisent des activités socio culturelles (évènements culturels et musicaux par exemple) qui n'ont pas pour seul objectif de renforcer les liens entre ses membres, mais aussi de trouver de l'argent pour conduire des projets solidaires dans les villages d'origine (amélioration des infrastructures, projets sociaux pour les personnes âgées, etc). La participation à ces activités et l'adhésion à ces fondations n'est pas limitée aux immigrés et réfugiés résidant en Suisse, mais s'étend à d'autres immigrés des mêmes villages dans d'autres pays d'accueil.

\section{Activités de loisir}

D'après les experts interrogés, quelques activités collectives de loisir, comme des mariages, deviennent transnationales. Les invités viennent de différents pays d'accueil et de Turquie, ils apportent de l'argent et/ou des cadeaux. La compagnie de télévision Dügün TV (Mariage Télé) diffuse des célébrations de mariages en Suisse, qui peuvent donc être regardées par d'autres membres de la famille et des amis dans le village d'origine. C'est un exemple montrant combien les activités de loisir deviennent transnationales. De la même façon, les évènements culturels (musique ou théâtre) organisés par les immigrés impliquent divers acteurs transnationaux (musiciens, coordinateurs) venant non seulement de Turquie mais aussi d'autres pays d'Europe.

\section{Activités intellectuelles}

Initiées surtout par d'anciens réfugiés, ces activités façonnent le contexte d'un échange d'idées entre immigrés et intellectuels en Turquie. Un certain nombre d'écrivains, de journalistes et de personnalités politiques qui vivent au pays d'origine ou dans d'autres pays d'accueil sont régulièrement invités à parler à des conférences, ou à prendre part à des débats organisés en Suisse.

4 http://www.vereinbasakschweiz.ch/engagement/mitgliedschaft.shtml, 25.06.2006. 


\section{Activités religieuses}

Beaucoup d'activités socio culturelles sont liées à des activités religieuses. Elles constituent un champ qui n'est pas seulement religieux, mais aussi social, offrant aux immigrés, y compris la seconde génération, de nombreuses occasions d'entreprendre des activités transnationales. Elles sont très institutionnalisées. Certaines associations religieuses comme l'Association islamique Milli Görüs et la Fédération des communautés Alévites, une minorité religieuse dans l'Islam, existent dans de nombreux pays européens et ont déjà formé des structures transnationales qui fonctionnent entre pays d'origine et pays d'accueil. Leurs membres paraissent faire preuve d'une forte allégeance à la même communauté, quel que soit le pays où ils vivent.

C'est un domaine d'activité où l'État turc représente un acteur important, qui stimule les activités religieuses transnationales. En tant qu'institution publique du gouvernement turc, le Diyanet est aussi présent en Suisse comme dans tous les pays d'accueil, et il est responsables des imams et des enseignants religieux envoyés de Turquie. Dans toute l'Europe, Diyanet organise des pèlerinages à la Mecque et le sacrifice rituel d'animaux durant les Fêtes islamiques pour le compte des immigrés. Elle lève.des fonds dans différents pays d'accueil, achète dans d'autres pays (Bosnie Herzégovine) des moutons qui seront distribués à des Musulmans dans le besoin. Cette activité couvre plusieurs pays.

De plus, les structures religieuses (mosquées, par exemple) ne servent pas seulement des buts religieux, mais aussi des activités sociales transnationales telles que des réunions ou des conférences avec des personnes venant d'autres pays. Les experts interrogés s'accordent pour dire que les activités religieuses transnationales attirent beaucoup la seconde génération en Suisse. Ces derniers occupent des positions importantes comme membres actifs ou organisateurs des associations religieuses transnationales.

Tableau 2 : Activités socio-culturelles des immigrés en Suisse

\begin{tabular}{|l|l|}
\hline Catégories & Pratiques \\
\hline Activités quotidiennes & $\begin{array}{l}\text { Lecture de journaux, télévision, radio, internet, communication, } \\
\text { etc. dans la langue d'origine }\end{array}$ \\
\hline Activités caritatives & $\begin{array}{l}\text { Soutien aux activités caritatives dans le pays d'origine, au village } \\
\text { d'origine par des moyens financiers, logistiques, formation, etc. } \\
\text { Fondations institutionnalisées avec ces buts }\end{array}$ \\
\hline Activités de loisir & $\begin{array}{l}\text { Fêtes de mariage } \\
\text { Évènements culturels (musique, théâtre) }\end{array}$ \\
\hline Activités intellectuelles & $\begin{array}{l}\text { Organisation d'évènements permettant les échanges d'idées entre } \\
\text { immigrés et intellectuels d'origine turque vivant hors de Turquie }\end{array}$ \\
\hline Activités religieuses & $\begin{array}{l}\text { Mise en place d'organisations ou d'associations religieuses } \\
\text { Organisation de pèlerinages à la Mecque } \\
\text { Collecte de fonds pour le sacrifice d'animaux dans le pays } \\
\text { d'origine ou un autre pays pendant les Fêtes islamiques }\end{array}$ \\
\hline
\end{tabular}


Il faut faire deux observations importantes sur les activités socio culturelles des immigrés mentionnées au tableau 2. D'abord, les immigrés turcs font preuve d'une grande propension à institutionnaliser leurs activités. D'habitude, les structures institutionnelles d'une fondation prennent la place d'associations précédentes, en tant que structure dominante pour les activités sociales et culturelles. Selon nos experts et acteurs, cette préférence tient aussi au fait que la politisation des associations socio culturelles d'immigrés au cours des années 1980 a produit un certain nombre d'effets négatifs ainsi qu'un scepticisme général parmi les immigrés turcs envers les activités organisées par ces associations. Une autre raison d'institutionnalisation tient à la nature de plus en plus financière des activités caritatives et de solidarité, un secteur en expansion qui requiert la responsabilité légale et professionnelle de sa direction. L'institutionnalisation des activités sociales apporte une solution à ces contraintes.

En second lieu, l'organisation d'activités culturelles transnationales comme la musique ou le théâtre devient de plus en plus commerciale. Les associations culturelles traditionnelles d'immigrés, en charge à l'origine de programmer ces évènements, ont été remplacées par des organisations professionnalisées. Cette « commercialisation de la sphère culturelle » peut aussi être observée dans quelques activités sociales traditionnelles, telles que les fêtes de mariage, comme nous l'avons déjà vu à propos de la compagnie TV Dügün en Allemagne. Il en résulte que l'implication des milieux économiques dans les activités socio culturelles des immigrés diversifiera sans nul doute les types d'activités disponibles et renforcera la dimension transnationale de ces activités dans l'avenir.

\section{Motivations et accès aux activités}

Le maintien des liens avec le pays d'origine représente une motivation essentielle des pratiques des immigrés turcs dans le domaine social. La création de ces liens est très importante pour la première génération et justifiée par un certain nombre de constantes sociologiques telles que vivre selon les traditions culturelles et sociales, se tenir informé des évènements en Turquie (lire les journaux turcs, regarder la télé et écouter la radio, regarder des sites turcs sur internet), garder un sentiment de solidarité avec la famille, les voisins et les amis au pays, et finalement, avoir un projet individuel de retour. De plus, quelques activités transnationales ont des motivations intellectuelles. De fait, beaucoup d'immigrés de la seconde génération se considèrent comme des acteurs sociaux qui utilisent les activités sociales transnationales pour aider à leur émancipation sociale. Comme le dit Kémal :

«La raison principale qui fait que je me suis impliqué dans ces activités sociales fut que je devais faire quelque chose, me voir comme acteur de ma vie sociale, et bâtir un réseau social par moi-même. »

Plusieurs immigrés de la seconde génération jouent un rôle important dans la direction de ces associations sociales. Pour Kémal, qui est responsable d'une fondation caritative :

"Ils s' intéressent aux actions de solidarité de notre fondation. Leur motivation ne repose pas sur leurs relations avec le pays d'origine de leurs parents, mais plutôt sur le fait qu'ils se donnent à eux-mêmes la possibilité d'être des acteurs sociaux conscients en Suisse. » 
De même, pour une autre personne interrogée :

« La participation de la seconde génération à nos activités est motivée d'abord par leur intérêt pour des problèmes qui les concerne directement, tels que les discriminations, les problèmes identitaires et le racisme. Ces personnes cherchent des lieux qui leur permettent de s'exprimer librement. La Bibliothèque Mozaic fournit un tel lieu pour des activités intellectuelles et artistiques. Donc, la seconde génération est beaucoup plus intéressée par les activités bilingues (turc et allemand). »

La possibilité de commercialiser certains types d'activité sociale est une autre motivation pour certains immigrés. Il existe plusieurs organisateurs individuels, ou de petites compagnies qui organisent des soirées de détente (musique ou théâtre avec des artistes venus de Turquie) pour satisfaire l'intérêt des Turcs en Suisse. Cette facilitation de la culture peut aussi être observée dans le domaine de l'information et des médias. Les organisateurs de ce type d'activités transnationales affirment répondre aux besoins sociaux des immigrés, comme le dit Kadir, éditeur d'un journal turc en Suisse :

"La communauté turque en Suisse veut avoir des informations sur les problèmes liés aux assurances, aux lois sur les migrations ou d'autres problèmes administratifs et légaux. Notre activité dans les médias répond à ces besoins. »

Beaucoup d'immigrés mettent leur émancipation sociale en Suisse en rapport avec ces activités transnationales qui, selon les personnes interrogées dans la sphère sociale, ne feront que croître dans l'avenir.

\section{Le caractère transnational des activités}

Les activités sociales turques parmi les immigrés sont de plus en plus conçues et organisées de façon transnationale par la collaboration de différents acteurs dans plusieurs pays de destination. Afin de donner une image plus exacte du caractère transnational de ces activités, regardons de plus près la dynamique d'un mariage turc en Suisse. L'animation musicale du mariage est souvent déléguée à un groupe musical basé en Allemagne, et l'organisation culinaire est confiée à une entreprise de restauration en France. Les invités arrivent de différents pays européens d'accueil et de Turquie. À la demande, un mariage peut être diffusé par satellite, de façon que les membres de la famille qui ne peuvent assister à la cérémonie en Suisse puissent voir le déroulement des évènements en direct, devant leur télévision, chez eux. Cela est rendu possible grâce à une compagnie de TV privée (TV Mariage) basée en Allemagne, qui diffuse la célébration en direct. Les mariages permettent aussi aux jeunes habitant dans différents pays de se rencontrer et quelquefois de trouver leur futur partenaire. En outre, d'autres activités, plus intellectuelles, permettent aux immigrés d'être en contact avec le monde intellectuel et artistique turc, comme le dit Kémal :

"Nos invités ne viennent pas seulement de Turquie. Nous invitons aussi des gens qui vivent dans d'autres pays d'accueil, spécialement l'Allemagne, où existe une atmosphère intellectuelle très développée, ce qui est très important pour la seconde génération. " 
En général, les immigrés turcs et leurs associations organisent des « soirées » (gece). C'est une façon collective de profiter de congés spéciaux (célébrations politiques, religieuses et sportives). Ces « soirées » sont aussi la base d'activités populaires fondées sur la solidarité et le caritatif. Une fondation de village, par exemple, organise une "soirée» destinée à rassembler les immigrés venus du même village ou ville et qui vivent à l'étranger. Elles prennent place dans un pays d'accueil différent chaque année et les invités viennent de toute l'Europe. L'argent collecté durant ces « soirées » est envoyé en Turquie, afin de contribuer à l'amélioration des infrastructures des villages et villes d'origine des immigrés.

\section{PRATIQUES POLITIQUES TRANSNATIONALES}

Depuis plus d'un demi-siècle, la Turquie a connu un système démocratique parlementaire multipartite, qui n'a pu, jusqu'à présent, intégrer toute la diversité politique et idéologique du pays. Jusqu'aux années 1990, il était interdit d'appartenir à un parti communiste ; ainsi, avant cette date, de nombreuses personnes furent emprisonnées ou forcées à l'exil. Les activités politiques ayant un arrière-fond ethnique ou religieux étaient également prohibées. S'identifiant fortement avec le modèle républicain français, l'État centralisateur turc rencontre encore des difficultés à répondre sur un mode libéral aux revendications politiques qui suivent des clivages idéologiques, ethniques et religieux s'écartant du modèle républicain officiel. Dans l'histoire démocratique des 50 dernières années, la Turquie a connu trois interventions militaires qui ont, en conséquence, banni à chaque fois, par la force, toute opposition politique.

En conséquence, le principal modèle du système politique turc est façonné par la présence de mouvements politiques et idéologiques extrêmement conflictuels basés sur une culture politique qui favorise l'action hors normes, parfois violente (Tachau et Heper, 1983). Cette culture politique conflictuelle a contribué à créer une politisation hautement polarisée des citoyens turcs, en Turquie et à l'étranger. Les immigrés turcs en Suisse, comme dans d'autres pays d'accueil, ont construit un certain nombre d'organisations politiques et sont impliqués dans diverses activités politiques qui offrent une plateforme non seulement à la génération plus âgée, mais aussi à la seconde génération (Vermeulen, 2006). Pourtant, l'appréciation de ces activités est souvent ambiguë, dans le pays d'origine comme dans la société d'accueil. Pour cette dernière, l'identification d'un immigré avec les objectifs et la culture politique du pays d'accueil est considérée comme un indicateur d'intégration politique. Bien entendu, l'engagement politique des immigrés dépend aussi des opportunités qui sont ou non offertes à la communauté immigrée par le pays d'origine (Ostergaard-Nielsen, 2001). Mais nous en savons assez peu sur la dimension transnationale des activités politiques des Turcs en Suisse.

\section{Description et types d'activité}

Les activités politiques des immigrés et réfugiés turcs en Suisse se caractérisent par une grande variété d'affinités politiques et idéologiques (gauche, droite, islamique, marxiste, etc.), de formes d'organisation (associations, partis et structures ad 
hoc) et de modèles de participation (conventionnels ou non). Afin de mieux comprendre la dimension transnationale de ces activités, nous classerons les pratiques en deux catégories ; a) celles qui sont d'abord orientées vers le pays d'origine ; b) celles qui s'orientent vers le pays d'accueil.

\section{Activités en direction du pays d'origine}

Dans cette catégorie, les pratiques les plus conventionnelles concernent le vote aux élections nationales. Pourtant, les citoyens turcs ne peuvent voter à l'étranger, ils ne peuvent le faire qu'à la frontière ou dans les aéroports turcs. La communauté turque s'arrange cependant pour soutenir ses mouvements ou partis politiques en affrétant des vols et des autobus là où il est possible de voter. Cette implication des immigrés turcs ne relève pas d'une simple stratégie de marketing politique : en fait les immigrés paient eux-mêmes toutes leurs dépenses de voyage. Ce qui encourage surtout ces activités, c'est d'abord une forte identification aux enjeux politiques du pays d'origine. Elle découle aussi de l'implication active dans les associations politiques mises en place par les « entrepreneurs politiques » de la communauté des immigrés et réfugiés en Suisse.

De plus, les associations et organisations politiques qui s'identifient fortement à des enjeux idéologiques, ethniques ou religieux en Turquie ont tendance à développer des liens institutionnels avec les organisations politiques dont ils se sentent proches. Il arrive souvent qu'une organisation politique turque crée une branche ou une association de soutien à l'étranger. Celles qui se sont créées en Europe constituent la « branche européenne » de l'organisme de base. La direction de cette branche se trouve généralement en Allemagne, d'où sont planifiées et dirigées les activités politiques transnationales. Les différentes branches locales et nationales des pays d'accueil assistent les organismes de base en fournissant des supporters, en aidant à la collecte de fonds, et aussi en apportant de l'information sur l'opinion publique à l'étranger. Ils publient des journaux politiques et organisent des «soirées » politiques (gece), avec musique et programme culinaire, auxquels sont invités des personnages politiques turcs.

Les activités transnationales des immigrés et réfugiés turcs n'ont pas toujours des objectifs à long terme. Les immigrés organisent parfois des activités collectives ad $h o c$, spécifiques à un moment et un lieu, qui sont indépendants de toute tendance idéologique, politique ou religieuse en Turquie. Le rôle de ces activités est d'exercer une influence politique sur ceux qui mettent en place les politiques locales ou nationales en Turquie. Ils sont souvent centrés sur des objectifs humanitaires et/ou environnementaux dans le village ou la ville d'origine. Ceux qui vivent dans d'autres pays d'accueil en Europe s'impliquent également dans ces activités. Par exemple, un certain nombre d'immigrés venant de la région de Batman et vivant dans divers pays européens ont été mobilisés contre la politique gouvernementale turque favorable à la construction d'une digue près de la ville de Hasankeyf, un site historique ancien.

Les activités orientées vers le pays d'origine n'excluent pas l'implication dans les enjeux politiques du pays d'accueil, mais le plus souvent de façon indirecte. En fait, les organisations et associations turques établissent des liens avec les acteurs politiques du pays d'accueil, spécialement avec les partis politiques (en général de gauche ou verts), les syndicats et les organisations de défense des immigrés. Ils participent aux 
activités politiques qui ont pour but d'améliorer les conditions de vie des immigrés, de les doter de droits politiques et de dénoncer les discriminations et le racisme. Selon un expert, ces actions politiques transnationales créent un dialogue entre les Turcs militants et des acteurs des organisations politiques du pays d'accueil. C'est le résultat d'une stratégie instrumentale, car les immigrés concernés cherchent le plus souvent de l'aide pour soutenir leur cause politique en Turquie plutôt qu'ils ne cherchent à s'identifier à un objectif politique du pays d'accueil.

\section{Activités politiques en direction du pays d'accueil}

Un grand nombre d'immigrés et de réfugiés turcs s'identifient aux enjeux politiques du pays d'accueil et s'impliquent dans diverses formes d'activité politique. Selon les experts interrogés, quelques Turcs de la première génération, en particulier des immigrés naturalisés, et des membres de la seconde génération, sont impliqués dans des enjeux suisses, via leur adhésion à des partis politiques locaux, surtout de gauche (sociaux-démocrates et verts), mais aussi, et de plus en plus, dans des partis de centre droit. De même, plusieurs immigrés turcs naturalisés préfèrent la participation institutionnelle, en participant aux instances municipales et aux comités consultatifs comme le Ausländerbeirat, ou les commissions d'intégration.

De plus, quelques organisations d'immigrés se rassemblent autour de certains enjeux politiques dans le pays d'accueil. Ils sont surtout actifs dans des organismes de défense des droits des immigrés (Forum pour l'intégration des migrants et immigrants, ou FIMM, les syndicats et des organismes de défense des réfugiés), qui aident à l'amélioration de leurs conditions de vie en Suisse. Pour les organisations d'immigrés turcs et kurdes, les enjeux de l'immigration sont cruciaux dans le processus d'obtention de la coopération des organismes suisses à la défense des immigrés. Tel est le cas de l'organisation politique Fédération des Travailleurs Démocrates (DIDF) ${ }^{5}$.

Tableau 3 : Activités politiques des immigrés turcs en Suisse

\begin{tabular}{|l|l|}
\hline Catégories & Pratiques \\
\hline Orientées vers le pays d'origine & Participation traditionnelle : vote individuel aux élections \\
& nationales \\
& Implication dans l'entreprenariat \\
& Politique : partis politiques organisations, associations et \\
& mouvements \\
& Activités ad hoc : mobilisation collective pour des causes \\
& humanitaires et/ou environnementales \\
\hline Orientées vers le pays d'accueil & Participation institutionnelle aux instances municipales ou \\
& aux conseils consultatifs locaux \\
& Implication dans des organismes suisses de défense des \\
& droits des immigrés \\
& Adhésion à des partis politiques suisses \\
\hline
\end{tabular}

5 Cette fédération (DIDF), créée en 1980 en Allemagne par des réfugiés, remonte à un mouvement turc marxiste, qui possède de nombreuses branches dans différents pays d'accueil. Ses activités sont dirigées surtout vers le pays d'accueil, et en partie seulement vers la Turquie (voir www.didf.ch, de juin 2007). 
Même si l'on peut caractériser les activités politiques des immigrés turcs selon leur orientation première, c'est à dire vers le pays d'origine ou vers le pays d'accueil, aucune de ces catégories ne fonctionne seule, ce qui favorise diverses formes d'activités politiques transnationales.

\section{Motivations et accès aux activités}

Selon les experts interrogés et les acteurs politiques, il existe plusieurs motivations qui stimulent les Turcs de Suisse à participer à des activités politiques transnationales. La plus répandue est l'identification idéologique ou religieuse à un mouvement ou un parti dans le pays d'origine. Immigrés turcs et kurdes importent avec eux leurs enjeux idéologiques et religieux et organisent leurs activités politiques en Suisse autour de ces enjeux. Pour la majorité des anciens réfugiés (marxistes ou alternatifs de gauche) l'idéologie est le principal facteur de mobilisation de leurs activités transnationales. En même temps les croyances religieuses paraissent être des motivations importantes pour la première génération, spécialement lorsqu'il s'agit de travailleurs manuels et de leurs enfants.

De même, l'identification à un groupe ethnique en Turquie constitue un autre stimulant à l'engagement politique transnational, en particulier concernant les activistes kurdes qui soutiennent la cause kurde en Turquie. L'opinion d'un de nos experts est révélatrice :

"Pour pratiquer en permanence une politique orientée vers la Turquie, il est nécessaire d'avoir de très fortes raisons, ethniques, religieuses ou idéologiques. Si ce n'est pas le cas, il n'est ni probable ni réaliste de conserver à long terme un lien politique avec la Turquie. » (Z.Y.)

Il importe de noter que la Suisse joue un rôle dans les activités politiques transnationales des activistes turcs et kurdes. De fait, l'environnement politique dans lequel prennent place les activités politiques des immigrés constitue un cadre important de leur socialisation politique, spécialement lorsqu'il s'agit des responsables de ces organisations et associations. Être en contact, organiser des rencontres communes, négocier des stratégies de collaboration avec les acteurs politiques suisses les aide à se socialiser à la culture politique locale (en particulier un modèle consensuel de bonnes relations entre partis et autonomie politique des groupes).

Dans ce contexte, le désir de transférer au pays d'origine l'expérience politique acquise en Suisse représente une forte motivation pour les acteurs politiques transnationaux. Leur prise de contacts avec les acteurs politiques turcs (en général ceux qu'ils connaissent déjà ou qui appartiennent à un parti ou mouvement dont ils se sentent proches) a pour but d'échanger expériences et connaissances. Il est intéressant de noter que les membres politiquement actifs de la seconde génération justifient leurs activités politiques transnationales par des arguments semblables. Parfois, cette motivation exprime un désir implicite de ressourcer leur activité à travers la vie politique en Turquie. Cela implique la possibilité de retourner au pays avec l'objectif d'entrer 
dans l'arène politique turque comme représentant au niveau local ou national. Comme le dit un expert interrogé, qui est également membre d'une instance municipale en Suisse :

"J'aimerais m'impliquer politiquement en Turquie afin de pouvoir transférer dans mon pays la culture politique que j'ai acquise ici. Ici, nous faisons une politique basée sur l'objectif concret (Sachlichkeit), ce qui n'est pas le cas en Turquie. Je pense que ce serait une contribution importante à la politique en Turquie. »(H.S.)

Pour certains acteurs politiques de la première génération, spécialement les réfugiés, la socialisation politique dans le pays d'accueil peut être une motivation importante, basée sur une identification avec les enjeux politiques en Suisse plus nette qu'avec les enjeux turcs. Cette tendance est plus affirmée lorsqu'elle s'accompagne d'une désillusion quant à la politique du pays d'origine. Selon le même expert :

"Les activités politiques à destination du pays d'origine disparaissent après un certain temps, provoquant des désillusions politiques parmi les militants et les cadres. Afin de contrer ces désillusions, au niveau individuel, quelques dirigeants, qui ont encore la capacité et les ressources pour agir sur le plan politique, investissent plus dans la politique locale du pays d'accueil. »

Selon les experts, l'engagement politique dans ce type de situation résulte de «stratégies purement individuelles et pas du tout collectives ». Au-delà de souhaits et désirs personnels, conditions sine qua non de l'engagement politique, ces explications montrent que différentes motivations peuvent coexister pour lancer une activité politique transnationale. Gül, ancien réfugié politique naturalisé, est arrivée en Suisse il y a 25 ans, à l'âge de 23 ans. Membre de la gauche, elle siège dans un Parlement cantonal. Elle souligne la coexistence de diverses motivations, tout en parlant de sa propre situation :

"Mon passé, les relations politiques en Turquie et mon attachement idéologique à la Gauche turque sont les principales raisons de mon entrée en politique. Pour ce qui concerne mon engagement présent dans la vie politique suisse, je voulais avoir une existence politique, et pas seulement économique dans mon nouveau pays ».

Fatma, activiste naturalisée, qui travaille dans une organisation turque, et est aussi un membre actif du parti vert, représente son organisation au Forum pour l'intégration des immigrés (FIMM). Elle confirme l'affirmation ci-dessus de son compatriote :

"Lorsque je m'implique dans des activités politiques en relation avec la Turquie, je satisfais mon besoin de solidarité envers un pays où j'ai vécu et entrepris ma socialisation. Mais, pour améliorer nos conditions de vie, en tant qu'immigrés en Suisse, ce me paraît être un vrai devoir que de participer à la vie politique ici. »

Pour Nilüfer, étudiante de 24 ans à l'université, et également membre d'un Parlement cantonal, arrivée en Suisse avec sa famille réfugiée, alors qu'elle avait 10 ans, la motivation de son engagement politique ne se limite pas à l'amélioration des 
conditions de vie des immigrés. Elle veut un changement social général dans la société. De plus, son aspiration à développer des activités de relais entre sa communauté d'origine (Kurde Alevi) et les Suisses d'origine est beaucoup plus importante que son engagement dans des activités dirigées vers la Turquie :

"J'aimerais améliorer les conditions de vie de tout le monde, les immigrés et les Suisses, sans aucune distinction. D'autre part, j'essaie aussi de créer des ponts entre ma communauté d' origine et la communauté suisse. Mon but est de mettre en place des projets communs entre ces deux communautés, qui constituent ma réalité et la source de mon identité. »

À l'évidence, intérêts et motivations individuels ne suffisent pas à expliquer un engagement politique. L'individu motivé doit avoir des ressources qui lui permettent de réaliser ses ambitions politiques. Dans un contexte migratoire, l'accès aux structures politiques du pays hôte est fortement conditionné par la disponibilité de ressources humaines, comme l'indique un autre acteur politique :

"Ce ne sont pas seulement mes capacités linguistiques et ma connaissance du système politique suisse et de ses institutions qui m'ont aidé à m' impliquer dans la vie politique suisse, mais aussi mes contacts sociaux et politiques, ainsi que le fait que je sois marié à une femme suisse. »

Au-delà de ces ressources individuelles en termes de compétences sociales, qui aident les immigrés à s'identifier aux enjeux politiques du pays hôte, les immigrés qui ont pour but de participer aux institutions politiques, dans les Parlements régional ou national par exemple, doivent posséder la nationalité suisse pour pouvoir être élus, sauf dans quelques municipalités qui rendent les immigrés éligibles.

L'attitude des entrepreneurs politiques du pays hôte peut aussi stimuler ou freiner l'identification des immigrés aux enjeux politiques. Les partis suisses font preuve d'une « ouverture modeste » — pour paraphraser un des acteurs interrogés — à l'intégration d'immigrés dans leurs structures. Cela est dû au fait que plusieurs communautés d'immigrés représentent un certain potentiel électoral. Cette attitude nouvelle de quelques partis suisses facilite l'accès des immigrés à la scène politique autochtone, comme le fait remarquer l'un de nos interviewés :

«Les partis de gauche, notamment les Verts et les Socialistes, sont plus ouverts aux immigrés. Ce sont surtout les femmes qui leur donnent le plus d'appui. » (F.B.)

Pour ceux qui, au départ, s'orientaient plus vers les enjeux politiques dans le pays d'origine, s'identifier aux objectifs politiques dans le pays hôte signifie parfois « prendre conscience » de l'importance de ces nouvelles orientations, ce qui intervient, pour certains immigrés militants, après quelque temps de résidence dans le pays hôte Il faudrait plus d'informations pour estimer le moment exact et les causes de cette évolution. Sur ce point, les explications de deux acteurs, un ancien réfugié et une immigrée de première génération, sont importantes :

Gül : " au début de mon séjour en Suisse, je ne m'intéressais pas beaucoup aux activités touchant l'intégration des immigrés. Les évènements de Turquie me 
semblaient beaucoup plus importants. C'était une question de prise de conscience, mais aussi une phase politique que j' ai dû traverser. C'était aussi une question de temps. »

Fatma : "J'ai commencé à m'intéresser à la politique ici lorsque j’ai pris conscience du fait que je voulais avoir une action politique, ne pas être juste une travailleuse. Cela impliquait nécessairement l'acquisition d'informations sur ce pays et l'établissement de contacts avec des organisations et des associations suisses, ce qui, évidemment, prend du temps. »

Enfin, on peut noter que l'activité transnationale résulte de facteurs multiples, au niveau individuel et collectif, en interne comme à l'extérieur. De plus, s'identifier aux enjeux sociétaux et politiques paraît être une incitation forte à l'implication politique des immigrés.

\section{Le caractère transnational des activités}

Les personnes interrogées dont les activités s'orientent d'abord vers le pays d'accueil relèvent de divers modèles qui leur permettent cependant de conserver des liens politiques avec le pays d'origine. Ainsi, l'établissement de liens organiques avec des dirigeants politiques en Turquie (partis, mouvements politiques, organisations, etc), via la branche européenne de mouvements, partis ou organismes politiques du pays d'origine offre aux immigrés l'opportunité de s'impliquer dans des activités orientées vers le pays d'origine. Ce lien dénote un fort attachement idéologique, ethnique ou religieux aux enjeux politiques du pays d'origine et est surtout, mais non exclusivement, utilisé par des immigrés ou réfugiés de la première génération, comme Fatma :

"Le journal "Evrensel" ${ }^{6}$ est aussi publié en Europe. Il est, pour l' essentiel, imprimé en Allemagne : nous préparons seulement un supplément pour la Suisse. Je prends une part active à la préparation de ce journal. Grâce à lui, et aux manifestations politiques que nous organisons, je peux maintenir Je suis membre $d u$ DIDF. Nous rencontrons parfois d'autres branches du DIDF dans d'autres pays d'Europe pour discuter de nos activités futures. »

Le travail d'organisation avec des acteurs politiques dans le pays d'origine, bien que prenant du temps, est cohérent avec l'objectif de transférer des connaissances et des expériences politiques acquises dans le pays hôte :

"Je prends régulièrement part à la réunion festive annuelle en Turquie. Pendant ces rencontres, j'essaie de transmettre à mes amis l'expérience et la culture politiques que j' ai acquises en Suisse » (un interviewé)

Souvent, la participation à des activités politiques orientées vers le pays d'origine (manifestations, soirées politiques, débats), organisées avec leurs circonscriptions d'origine par des entrepreneurs politiques immigrés en Suisse constitue une autre façon de devenir acteur transnational. Comme le dit Gül, ces activités peuvent être planifiées et organisées par les acteurs eux-mêmes :

6 Evrensel, (L'Universaliste) est un quotidien publié en Turquie et idéologiquement proche du parti de gauche Emek Partisi (Parti du Travail). 
"J'utilise de façon réciproque les potentialités existantes. J'essaie de considérer quelques problèmes spécifiques concernant la Turquie (démocratisation, écologie) afin de gagner l'appui de l'opinion publique suisse. Pour y parvenir, j'organise des réunions d'information destinées aux cercles politiques suisses. »

Les acteurs transnationaux de seconde génération qui ne s'identifient pas aux enjeux idéologiques, ethniques ou religieux turcs recherchent des liens personnels avec des acteurs politiques dans le pays d'origine. Ils cherchent, comme le dit Nilüfer, à échanger des expériences politiques et à être informés de ce qui se passe en Turquie :

"Si je cherche à établir des contacts avec des politiciens en Turquie, ce n'est pas pour m' impliquer dans la vie politique turque. Mon but est d'entamer un échange d'idées et d'expériences. Je suis aussi très intéressée par ce que font les politiciens en Turquie, afin de comparer les faits et les enjeux. Mes relations politiques avec la Turquie se font par le biais des associations kurdes et alevis en Suisse, qui sont très impliquées dans les activités politiques en Turquie. Personnellement, je m'oriente vers la vie politique suisse.»

En dehors de ces formes non conventionnelles de relations politiques de nos acteurs transnationaux et binationaux, la participation aux élections nationales turques (dans les aéroports ou les bureaux de douanes) est également une participation à la transnationalisation de leurs pratiques politiques. Il est aussi important de noter que les partis, organisations et mouvements turcs cherchent à accéder aux ressources financières et politiques des immigrés. Ils ont presque tous créé des organisations qu'ils nomment « branches européennes », spécifiquement conçues pour servir les besoins des immigrés. Les acteurs politiques en Turquie reconnaissent que l'aide des immigrés à leurs propres structures politiques est importante et peut influer sur le vote des membres de la famille en Turquie, spécialement dans les zones rurales. Pour quelques mouvements politiques, tels que les organisations ethniques kurdes, l'intégration politique des immigrés dans le système suisse est perçu comme le signe d'un manque d'identification avec leur cause, la libération du Kurdistan. Ces organisations promeuvent de forts liens organisationnels avec les immigrés et leur engagement direct dans des activités dirigées vers le pays d'origine. D'autre part, comme pour certaines petites organisations d'immigrés turcs en Suisse, quelques mouvements ou partis encouragent l'implication dans la vie politique suisse, comme signe de bonne intégration. Ils souhaitent construire un réseau avec des représentants turcs en Suisse, comme cadre d'obtention des ressources financières et culturelles des immigrés, mais aussi pour accroître leur sphère d'influence.

\section{CONCLUSION}

Nous avons trouvé une grande diversité d'actions transnationales parmi les immigrés turcs en Suisse. Leur arrivée par vagues et par des voies légales différentes ont contribué au développement d'une variété de pratiques les plus adaptées aux différentes sphères, selon le statut, et la situation socio économique, culturelle et politique. Aucun argument raisonnable ne limite les activités transnationales à la première phase d'un processus migratoire. Au contraire, ces pratiques évoluent et 
atteignent leur sommet lorsque les immigrés ont déjà réalisé un certain niveau d'intégration et possèdent le capital social nécessaire à la création de liens aussi complexes. Les expériences transnationales des immigrés turcs montre qu'une bonne intégration n'exclut ni une forte identification avec le pays d'origine, ni le développement d'activités transnationales. Cela nous permet de dire que l'" identification transnationale » ne paraît pas, en général, être fonction de la position de l'immigré turc dans les structures du pays hôte. De même, une intégration réussie ne produit pas inévitablement un effet dissuasif sur une «identification transnationale ».

L'analyse des activités transnationales des immigrés turcs ne peut conforter l'hypothèse d'une explication par un fort intérêt à diriger le projet migratoire vers le retour au pays. Au contraire, la fonction des liens transnationaux peut être liée à l'instauration de meilleures conditions de vie et aux stratégies délibérées d'intégration des immigrés. Au niveau économique transnational, par exemple, les pratiques utilisées par les immigrés visent à contourner l'instabilité de leur position sur le marché du travail suisse, résultant d'un faible niveau d'éducation et de formation, mais aussi de discriminations.

Les activités transnationales des immigrés turcs ne dépendent pas nécessairement du type de migration (économique ou politique) : chaque sous-groupe tisse des liens transnationaux à la fois dans les domaines économique, politique et socio culturel. Cependant, le facteur générationnel semble être une variable explicative de certaines pratiques transnationales. Sur le plan politique, par exemple, la plupart des immigrés de première génération s'identifient fortement à des enjeux politiques du pays d'origine, alors que la seconde génération montre une nette tendance à s'identifier d'abord aux enjeux du pays hôte. Le facteur générationnel paraît agir aussi dans le champ social. Si la préservation de liens avec le pays d'origine est une motivation essentielle pour la première génération, un nombre important des immigrés de seconde génération utilise les activités sociales transnationales pour aider à leur émancipation sociale, en s'identifiant à des enjeux tels que la solidarité, les discriminations, l'identité et le racisme. Leurs ressources et capacités transculturelles paraissent être mieux reconnues dans un contexte transnational que dans le contexte national du pays hôte.

Enfin, il est important de montrer clairement que les activités transnationales ne se réduisent pas à l'espace géographique compris entre pays d'accueil et pays d'origine. Des pays tiers peuvent aussi jouer un rôle stimulant en renforçant les pratiques transnationales. C'est le rôle que paraît jouer l'Allemagne, qui représente une sorte de plaque tournante ou de quartier général pour les Turcs, ce qui réduit le rôle de la Turquie dans un certain nombre d'activités transnationales. Pour terminer, il faut insister sur la richesse de la forme et des contenus des pratiques transnationales des immigrés turcs. Nous avons tenté de saisir leurs principales dynamiques et caractéristiques, même si certains domaines, comme la santé et l'éducation, n'ont pu être abordées dans notre recherche. Mais ce qui ressort clairement est ce facteur social total : les pratiques transnationales transcendent l'importance de l'État nation, même si elles dépendent de facteurs nationaux spécifiques. 


\section{Références bibliographiques}

ARGAST Regula, ARLETTAZ Silvia et ARLETTAZ Gérald (2003) « Citoyenneté, nationalité et formation nationale en Suisse 1798-1925 », Études et sources, 29, pp. 129-160.

BACHMANN Carine et al. (eds) (2004) Emigration and return: photo stories of Armenian migrants, Geneva, Cimera.

D'AMATO Gianni (1997) "Gelebte Nation und Einwanderung: zur Trans-Nationalisierung von Nationalstaaten durch Immigrantenpolitik am Beispiel der Schweiz”, in Kleger Heinz (ed.) Transnationale Staatsbürgerschaft, Frankfurt, Campus Verlag, pp. 132-159.

D'AMATO Gianni und GERBER Brigitta (eds) (2005) Herausforderung Integration: städtische Migrationspolitik in der Schweiz und in Europa, Zürich, Seismo.

DAHINDEN Janine (2005) Prishtina - Schlieren: albanische Migrationsnetzwerke im transnationalen Raum, Zürich, Seismo.

FIBBI Rosita (2004) «L'approche transnationale dans l'étude des migrations », in Nedelcu Mihaela (ed.) La mobilité internationale des compétences : situations récentes, approches nouvelles: actes du colloque "La mobilité internationale des compétences : fuite ou circulation? » Neuchâtel, Paris, L'Harmattan, pp. 61-75.

FIBBI Rosita, KAYA Bülent et PIGUET Étienne (2003) Le passeport ou le diplôme ? Étude des discriminations à l'embauche des jeunes issus de la migration, Neuchâtel, Forum suisse pour l'étude des migrations et de la population.

FIBBI Rosita et MEYER Jean-Baptiste (2002) «Le lien plus que l'essence : introduction », in Fibbi Rosita et Jean-Baptiste Meyer (eds) Diasporas, développements et mondialisations, La Tour d'Aigues : Éditions de l'Aube, pp. 5-21.

GLICK SCHILLER Nina, BASCH Linda and BLANC-SZANTON Cristina (1995) "From immigrant to transmigrant: theorizing transnational migration", Anthropological quarterly, 68(1), pp. 48-63.

HANSEN Niles and CARDENAS Gilberto (1988) "Immigrant and native ethnic enterprises in Mexican American neighborhoods: differing perceptions of Mexican immigrant workers", International migration review, 22(2), pp. 226-242.

ØSTERGAARD-NIELSEN Kristine Eva (2001) "Transnational political practices and the receiving state: Turks and Kurds in Germany and the Netherlands." Global Networks, 1(3), pp. 261-281.

PIGUET Étienne (1999) Les migrations créatrices : étude de l'entreprenariat des étrangers en Suisse, Paris, L'Harmattan.

POLI Raffaele (2004) Les migrations internationales des footballeurs : trajectoires de joueurs camerounais en Suisse, Neuchâtel, Éditions CIES, Centre international d'étude du sport.

PORTES Alejandro (2001) "Introduction: the debates and significance of immigrant transnationalism", Global Networks, 1(3), pp. 181-193.

PORTES Alejandro, GUARNIZO Luis E and LANDOLT Patricia (1999) "The study of transnationalism: pitfalls and promise of an emergent research field", Ethnic and racial studies, 22(2), pp. 217-237.

RIAÑO Yvonne (2003) "Migration of skilled Latin American women to Switzerland and their struggle for integration", in Mutsuo Yamada (ed.) Emigración latinoamericana: comparación interregional entre América del Norte, Europa y Japón. Osaka: The Japan Center for Area Studies, National Museum of Ethnology, pp. 313-343.

TACHAU Frank and HEPER Metin (1983) "The State, Politics and the Military in Turkey", Comprative Politics, 16(1), pp. 17-33.

TRIBALAT Michèle (1995) Faire France : une grande enquête sur les immigrés et leurs enfants, Paris, Éd. La découverte. 
VAN AMERSFOORT Hans, MUUS Philip and PENNINX Rinus (1984) "International migration, the economic crisis and the state: an analysis of Mediterranean migration to Western Europe", Ethnic and racial studies, 7(2), pp. 238-268.

VERMEULEN Floris (2006) The immigrant organising process: Turkish organisations in Amsterdam and Berlin and Surinamese organisations in Amsterdam, 1960-2000. Amsterdam, Amsterdam University Press.

WICKER Hans-Rudolf (2001) "Swiss naturalization practices in times of accelerated transnational mobility and new racism: a preliminary research report", in Kletzander, Helmut and Wernhart Karl R (eds) Minderheiten in Österreich: kulturelle Identitäten und die politische Verantwortung der Ethnologie. Wien, WUV Universitätsverlag, pp. 125-14 


\section{a contrano}

revue interdisciplinaire de sciences sociales Vol. 5 - No 2

\section{NUMERO SPECIAL : LA PALESTINE \& LES CONFLITS DU MOYEN-ORIENT}

\section{Éditorial}

Articles

\author{
Daniel Meier \\ De la conflictualité au Moyen-Orient
}

Riccardo Bocco et Wassila Mansouri

Aide internationale et processus de paix : le cas palestinien, 1994-2006

Hanna Daoudi et Raja' Khalidi

The War-torn Palestinian Economy: Aid, Development

and State Formation

Jalal al-Husseini

Visions palestiniennes du «droit au retour » des réfugiés, sept ans après le début de la seconde Intifada (2000-2007)

Benoît Challand

Les mutations du leadership palestinien (1993-2007)

Pascal de Crousaz

Israël : la doctrine de " combat disséminé ». Vers une sur-violence sans rationalité militaire?

Rolf Schwarz

From Rentier State to Failed State : War and the De-Formation of the State in Iraq

Loulouwa al-Rashid et Edouard Méténier

A propos de la violence "irakienne». Quelques éléments de réflexion sur un lieu commun

Mohammed-Reza Djalili

La politique arabe de l'Iran

Mounia Bennani-Chraïbi

Les conflits du Moyen-Orient au miroir des communautés

imaginées : la rue arabe existe-t-elle ?Le cas du Maroc

Philippe Droz-Vincent

La décomposition régionale au Moyen-Orient : effets pervers

$d u$ «moment américain » 